\title{
Cycle évolutif
}

\section{de Galactosomum timondavidi Pearson et Prévot, 1971 \\ Trematoda : Heterophyidae}

\author{
Parasite du Goéland à pieds jaunes Larus argentatus
}

\author{
par Georges PREVOT \\ Université de Provence, Centre de Saint-Jérôme \\ Laboratoire de Biologie animale, F 13013 Marseille
}

\begin{abstract}
Résumé
L'auteur décrit et figure le cycle évolutif du Trématode Digène Hétérophyide Galactosomum timondavidi PEARson et PRÉvot, 1971. La cercaire se développe à partir de rédies chez le Gastéropode Prosobranche Cerithium mediterraneum. Elle pénètre chez différents Poissons et s'enkyste au niveau des lobes optiques de ces derniers. L'adulte se localise dans le tube digestif d'un Oiseau de mer: Larus argentatus.
\end{abstract}

\section{Summary}

The author describes and figures the life cycle of the Trematode Digenea Heterophyidae Galactosomum timondavidi PEARson and PrÉvot, 1971. The cercaria develops in rediae in the prosobranch gastropod Cerithium mediterraneum. It penetrates in various fishes and encysts in the brain of the latter. The adult is parasitic in the alimentary canal of a sea bird: Larus argentatus.

La réussite d'infestations expérimentales de poissons par des cercaires de Galactosomes nous a permis de reconstituer le cycle d'une espèce décrite précédemment sous le nom de Galactosomum timondavidi. Nous exposons ici le résultat de nos recherches. 
HôTE DÉFINITIF: Larus argentatus michaellis Naumann (Oiseau Laridé). Localisation: limite entre le deuxième et le troisième tiers intestinal.

PREMIER hôTE: Cerithium mediterraneum Deshayes (Mollusque Prosobranche), Localisation : hépato-pancréas.

Deuxièmes hôTes: Mugil auratus Risso, Syngnathus abaster Risso (Poissons Téléostéens). Localisation : cerveau.

Localisation GÉOgraphique: Port-Saint-Louis-du-Rhône, Bouches-du-Rhône, France.

\section{$1^{\circ}$ Rédies (fig. 1).}

Elles occupent, dans sa quasi-totalité, la glande digestive du Mollusque premier hôte ; leur pigmentation rougeâtre confère à l'hépato-pancréas une couleur bien caractéristique. Ces rédies ne présentent que de très faibles mouvements apparents. Leur taille varie entre $410 \times 80 \mu$ pour les petits exemplaires et $1100 \times 145 \mu$ pour ceux de grande taille; la moyenne se situe aux environs de $900 \times 140 \mu$.

Le pharynx, terminal, est subsphérique, légèrement plus large que long $(32-36 \times$ 33,5-37,5 $\mu$ ). Le sac digestif, extrêmement rudimentaire, mesure $12-14 \times 8,5-10 \mu$. L'orifice de ponte, souvent bien visible, s'ouvre à la limite postérieure du premier douzième de la longueur du corps. Chaque rédie renferme de nombreuses boules germinatives dont la taille va croissant depuis le fond de la rédie vers la région antérieure. En se rapprochant de l'orifice de ponte, les ébauches de cercaires apparaissent. A proximité de l'orifice de ponte, ces ébauches sont mieux différenciées mais n'atteignent jamais le stade de la cercaire mûre ; la plus grande taille observée alors est la suivante : $122 \times 46 \mu$ pour le corps de la cercaire et $180 \times 26 \mu$ pour la queue. Les cercaires sont donc «pondues » à un développement correspondant à la taille indiquée précédemment et achèvent leur maturation dans la glande digestive du Gastéropode dont les tissus renferment en effet de nombreuses cercaires très actives, à taches oculaires bien développées.

\section{$2 \circ$ Cercaire (fig. 2).}

La sortie des cercaires se fait de façon très massive et assez irrégulière. Le nombre de cercaires évacuées est à chaque fois très élevé, surtout lors des premières éme1gences (plusieurs milliers); leur durée de vie dans l'eau de mer est brève, elle ne dépasse pas 24 heures; la période d'activité, assez courte, atteint environ dix heures.

Ces larves, très sensibles à la lumière, se dirigent, dès la sortie de l'hôte, vers la zone d'éclairement le plus intense. Ce phototropisme est en relation avec la présence de deux volumineuses taches oculaires. Le déplacement des cercaires est réalisé par des ondulations de la queue constamment en mouvement et jouant un rôle tracteu. La vitesse moyenne de déplacement est de 0,2 centimètre par seconde. La morphologie de ces cercaires et leur sensibilité à la lumière laissent supposer qu'elles font partie du plancton marin. 

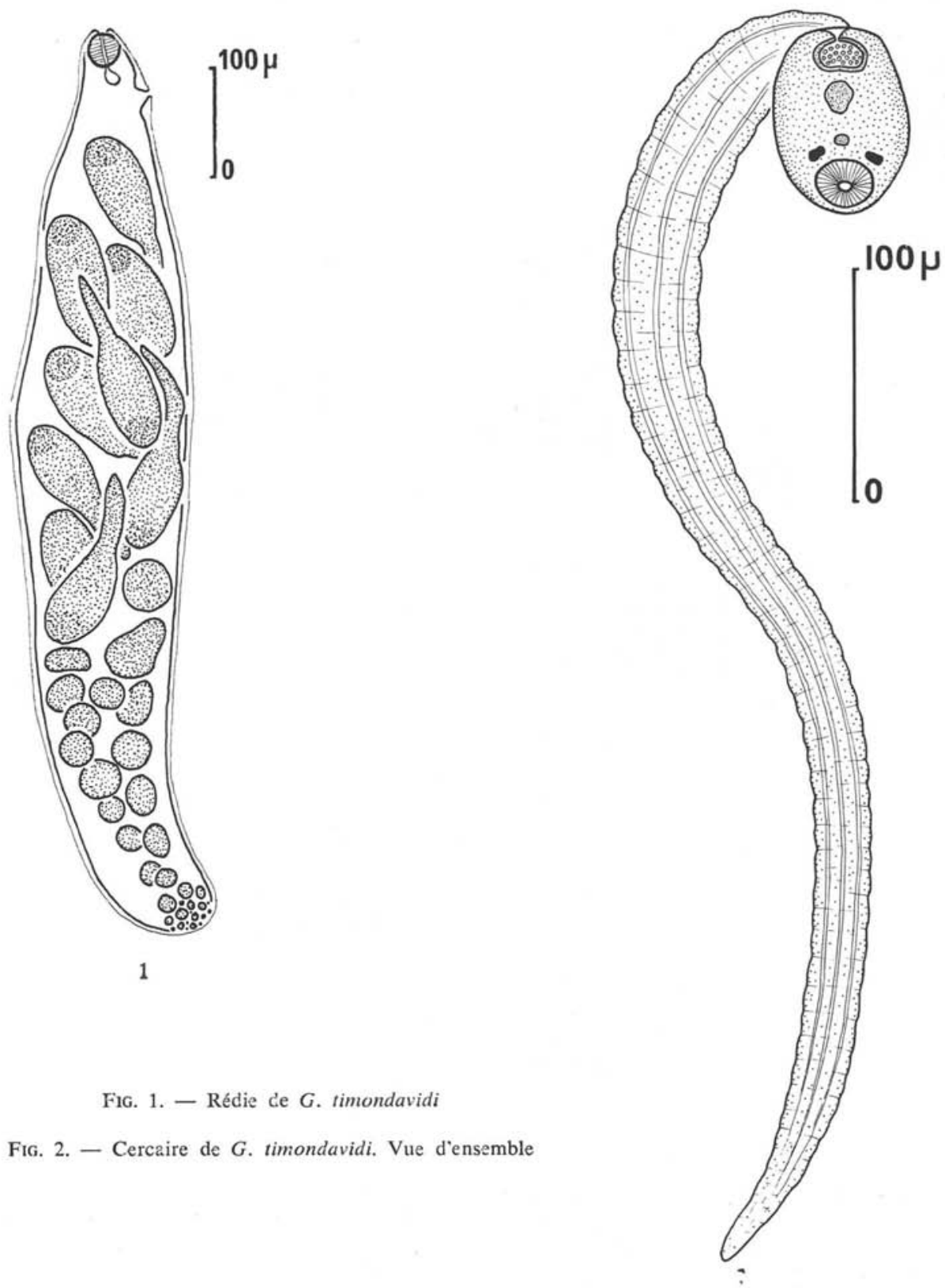

Fig. 1. - Rédie de G. timondavidi

Fig. 2. - Cercaire de G. timondavidi. Vue d'ensemble 
DESCRIPTION DE LA CERCAIRE MûRE (fig. 3).

- Corps : il est replié ventralement sur la queue; de forme sensiblement ovoïde, il est arrondi en avant et tronqué à son extrémité postérieure. Après fixation, coloration et montage, les larves gardent une taille sensiblement constante : $84-96 \times 52-64 \mu$ (moyenne : $88 \times 62 \mu)$. La cuticule est entièrement couverte de fines épines disposées en rangées régulières.

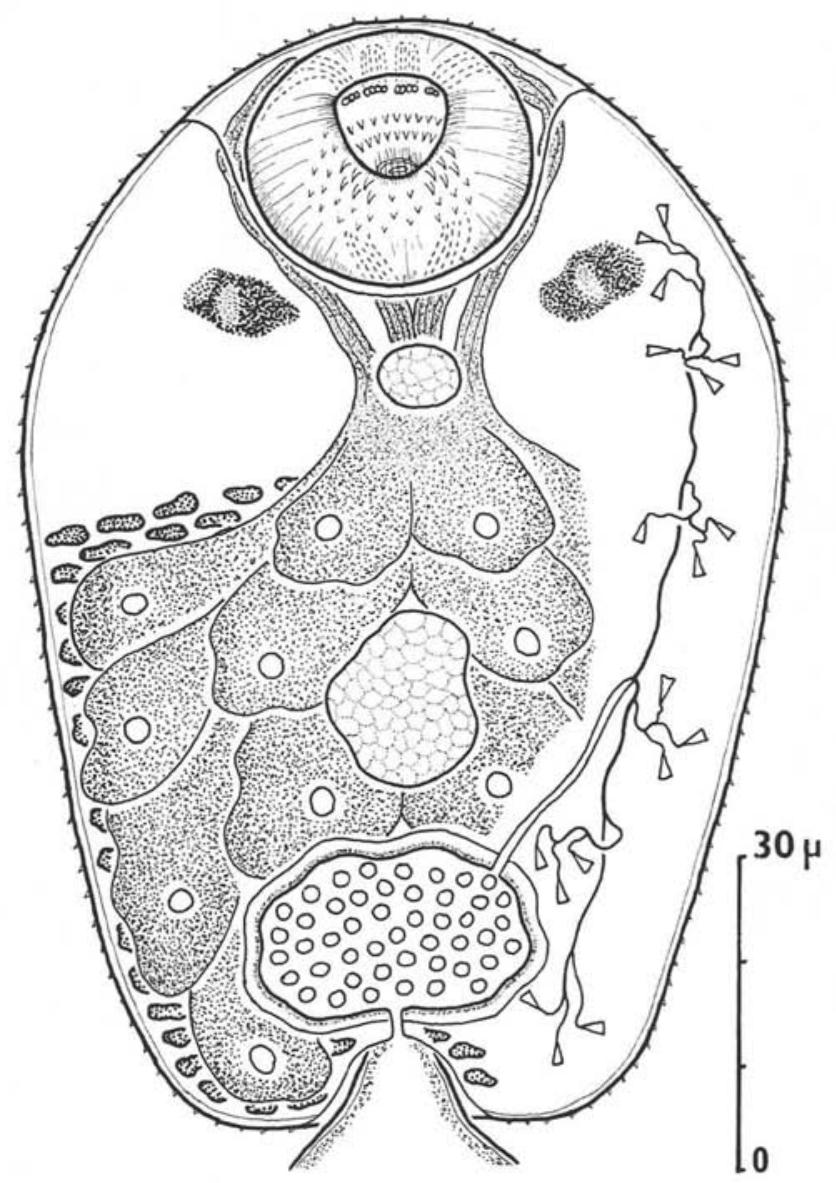

FIG. 3. - Corps de la cercaire de G. timondavidi. Vue ventrale

- Glandes de pénétration: sept paires de glandes volumineuses occupent la région postérieure à l'ébauche pharyngienne. Les canaux sécréteurs remontent antérieurement, passant entre les taches oculaires et l'ébauche pharyngienne. Deux groupes symétriques, constitués chacun par trois canaux externes, contournent la ventouse orale, deux autres groupes symétriques plus internes, constitués chacun par quatre canaux, passent dorso-latéralement à la ventouse. Tous ces canaux s'ouvrent au niveau de la lèvre supérieure de la ventouse orale. 
- Glandes cystogènes: elles se situent en face dorsale et s'étendent entre l'ébauche pharyngienne et la région postérieure du corps.

- Taches oculaires: volumineuses $(8,5 \times 6,5 \mu)$; disposées postéro-latéralement à la ventouse orale. Elles ont approximativement la forme d'un cylindre creux occupé au centre par le corps réfringent.

- Ventouse : c'est un organe protactile pourvu d'épines sur sa lèvre dorsale. En position de « repos», elle est ventrale et subterminale. Ses mensurations sur l'animal fixé restent très stables: 17,6-21,6 $\times$ 23,2-25,6 $\mu$ (moyenne: $19,2 \times 24 \mu$ ); sa hauteur est toujours légèrement inférieure à sa largeur. La lèvre dorsale, dans laquelle s'ouvrent les canaux des glandes de pénétration est armée de deux rangées de neuf épines de même taille $(1,5 \mu)$. L'orifice buccal, entouré d'une couronne de soies, s'ouvre au fond d'une crypte en forme d'entonnoir. Latéralement, sur le bord ventro-externe de l'entonnoir, sont disposés de façon symétrique deux groupes de six épines constitués chacun par deux rangées de trois épines. Un repli transversal ventral sépare la région de la ventouse orale du reste du corps. La ventouse orale n'est pas différenciée, ce sont des cercaires monostomes.

- Appareil digestif : il n'est pas encore apparent; seule est visible, en arrière des taches oculaires, une masse de $7 \times 9 \mu$ correspondant à l'ébauche du pharynx.

- Appareil génital: le primordium génital a l'aspect d'une masse irrégulière d'environ $16 \times 14 \mu$, visible en avant de la vessie.

- Appareil excréteur: il se compose de 36 protonéphridies arrangées en six groupes symétriques de trois et répondant à la formule : $2[(3+3+3)+(3+3+3)]=36$. Les cellules à flamme occupent essentiellement les régions latérales du corps. Elles sont disposées respectivement: à proximité des taches oculaires, immédiatement en arrière des taches oculaires, entre les taches oculaires et le primordium génital, au niveau du primordium génital, sur le bord antéro-latéral de la vessie, sur le bord postéro-latéral de la vessie. Les canaux excréteurs secondaires confluent à la hauteur du primordium génital pour donner les canaux collecteurs principaux de type mésostome; ces derniers débouchent dans une vessie de grande taille $(14 \times 27 \mu)$, de forme ovoïde et allongée transversalement. Les parois de la vessie sont épaisses, sa lumière est remplie de granulations sphériques de $1,5 \mu$ de diamètre. Le pore urinaire est ventral et subterminal.

- Queue: de très grande taille; ses mensurations varient avec l'état de contraction: $580-750 \times 48-35 \mu(640 \times 45 \mu$ en moyenne). Sa longueur est donc égale à 7 à 8 fois celle du corps de la cercaire. Elle est très légèrement pigmentée en rose, le pigment étant réparti de façon homogène.

\section{Discussion.}

Peu de cercaires à grande queue ont, jusqu'à maintenant, été signalées en Méditerranée. Ce sont : Cercaria clausii Monticelli, 1888 du Golfe de Naples, chez Trivia europaea, Cercaria equitator Sinitzin, 1911 de Cerithiolum exille de la Mer Noire, Cercaria mirabilicaudata Prévot, 1967 de Cerithium vulgatum de la région de Marseille.

Aucune de ces trois espèces n'est assimilable à la nôtre ; $C$. clausii étant une cercaire « roi des rats », les deux dernières étant d'une taille très nettement supérieure. 
La comparaison de notre espèce avec les cercaires de Miller et de Cable aux Etats-Unis montre qu'elle ne correspond à aucune des descriptions de ces auteurs. Il en est de même pour les cercaires à grande queue étudiées par Ito au Japon.

\section{$3^{\circ}$ Métacercaire.}

Nous l'avons obtenue expérimentalement en partant de cercaires. De petits muges (Mugil auratus) furent laissés en contact pendant quelques dizaines de minutes avec des cercaires à grande queue. Les poissons furent par la suite disséqués périodiquement. Nous avons ainsi trouvé la métacercaire dans le cerveau et suivi son développement jusqu'à la maturité.

Seize heures après l'infestation, les poissons renfermaient au niveau des lobes optiques de petits kystes ovoïdes à paroi hyaline, mesurant $44 \times 27 \mu$. La métacercaire jeune montre déjà une fragmentation des taches oculaires, les épines de la lèvre dorsale ne sont plus rangées régulièrement et parfois même se détachent.

Après 25 jours à $18^{\circ} \mathrm{C}$, le kyste mesure $200 \times 140 \mu$, la métacercaire $320 \mu$ de long pour $130 \mu$ de large. La ventouse orale a doublé de taille $(36 \times 42 \mu)$, la vessie est encore allongée transversalement $(40 \times 48 \mu)$. Le pharynx s'individualise $(36 \times$ $24 \mu$ ), de même que la ventouse génitale (diamètre de $20 \mu$ ). Les ébauches génitales sont encore indiscernables.

Après 35 jours à $18{ }^{\circ} \mathrm{C}$, la larve mesure $900 \mu$ de long et $50 \mu$ de large, la ventouse orale $75 \times 96 \mu$, le pharynx $65 \times 46 \mu$, la ventouse génitale approximativement $30 \mu$ de diamètre. En arrière de cette dernière, on peut distinguer une masse, correspondant à l'ensemble ovaire-testicules-réceptacle séminal, de $72 \times 48 \mu$. Toute la région postérieure est occupée par de grosses cellules masquant l'organisation ; il s'agit là de remaniements cellulaires qui aboutiront à la formation d'une vessie tubuleuse.

\section{MÉTACERCAIRE Mûre (fig. 4).}

Obtenue chez des muges parasités trois mois auparavant et maintenus dans de l'eau de mer à $18^{\circ} \mathrm{C}$.

Kyste de forme ovoïde, à paroi hyaline de $10 \mu$ d'épaisseur, mesurant $672-816 \times 480$ $600 \mu$. Il est entouré d'un xénokyste d'importance variable mais généralement peu développé. La larve est repliée sur elle-même dans la cavité kystique dont elle peut être aisément extraite. Sa forme est allongée (1940 à $2160 \mu)$; la largeur est sensiblement uniforme $(400$ à $430 \mu)$. Le tégument de $2,5 \mu$ d'épaisseur est recouvert d'épines dont la taille diminue progressivement en allant vers l'arrière. Quelques granules pigmentaires, restes des taches oculaires de la cercaire, sont encore visibles dans la région antéro-latérale du pharynx. Les cellules des glandes frontales sont disposées entre la bifurcation des caecums et le sac ventro-génital. La ventouse orale, terminale est moins haute que large (208-216 $\times$ 220-248 $\mu$ ), le prépharynx de taille variable $(60$ à $130 \mu)$, le pharynx puissant $(98-120 \times 78-85 \mu)$. Les caecums intestinaux, allongés, se terminent entre 70 et $120 \mu$ de l'extrémité postérieure.

La ventouse ventrale, un peu allongée longitudinalement, mesure 74-80 $\times$ 56-62 $\mu$. Le rapport des hauteurs ventouse orale sur ventouse ventrale est égal à 2,75 en moyenne. 
Appareil génital mâle: testicules en tandem; le testicule antérieur mesure $64-100 \times 80-130 \mu$; le postérieur 64-114 $\times$ 80-134 $\mu$. Les spermiductes confluent un peu avant d'atteindre la vésicule séminale; cette dernière mesure 170-180 $\times$ 80-84 $\mu$. Le canal éjaculateur s'ouvre dans l'atrium génital dorsalement par rapport à l'utérus.

Appareil génital femelle (fig. 5) : l'ovaire submédian se situe approximativement à mi-distance entre les extrémités du corps; il mesure $30-42 \times$ 46-62 $\mu$. Il s'en détache un oviducte aboutissant à un carrefour recevant d'une part le canal de Laurer et à l'opposé, en arrière de l'ovaire, le réceptacle séminal $(46-66 \times 80-100 \mu)$. Après ce carrefour, l'utérus se dilate; c'est à la hauteur de cette dilatation que s'abouche le vitelloducte impair très allongé, se bifurquant vers l'arrière entre les deux testicules. L'ootype fait suite au vitelloducte impair; l'utérus décrit vers l'arrière un certain nombre de boucles n'atteignant pas l'extrémité postérieure mais recouvrant la partie antérieure de la vessie.

Sac ventrogénital (fig. 6): médian, il se situe aux environs du premier tiers de la longueur du corps. Il enserre étroitement le gonotyl et la portion interne de la ventouse génitale. L'orifice du sac ventro-génital, ventral, s'ouvre généralement dans sa région postérieure; la paroi postérieure droite est pourvue de plusieurs rangées de petites épines de $1,2 \mu$ de long. Le gonotyl est bilobé, il mesure dans son ensemble 28-36 $\times 20-28 \mu$. Le lobe dorsal symétrique et musculeux $19 \times$ $17 \mu$, le lobe ventral asymétrique et peu musculeux $32 \times 18 \mu$. Le pore génital s'ouvre entre et à l'arrière des lobes du gonotyl.

Appareil excréteur (fig. 7): protonéphridies et canaux excréteurs sont bien visibles, mais en raison de leur grand nombre et de leurs intrications, nous n'avons pas réussi à établir la formule excrétrice.

Il y a six ensembles symétriques, chacun paraissant constitué par neuf cellules excrétrices. Les trois ensembles postérieurs se jettent dans un canal secondaire remontant jusqu'à la hauteur du pharynx; à ce niveau, le canal décrit une boucle rétrograde et conflue avec le canal secondaire

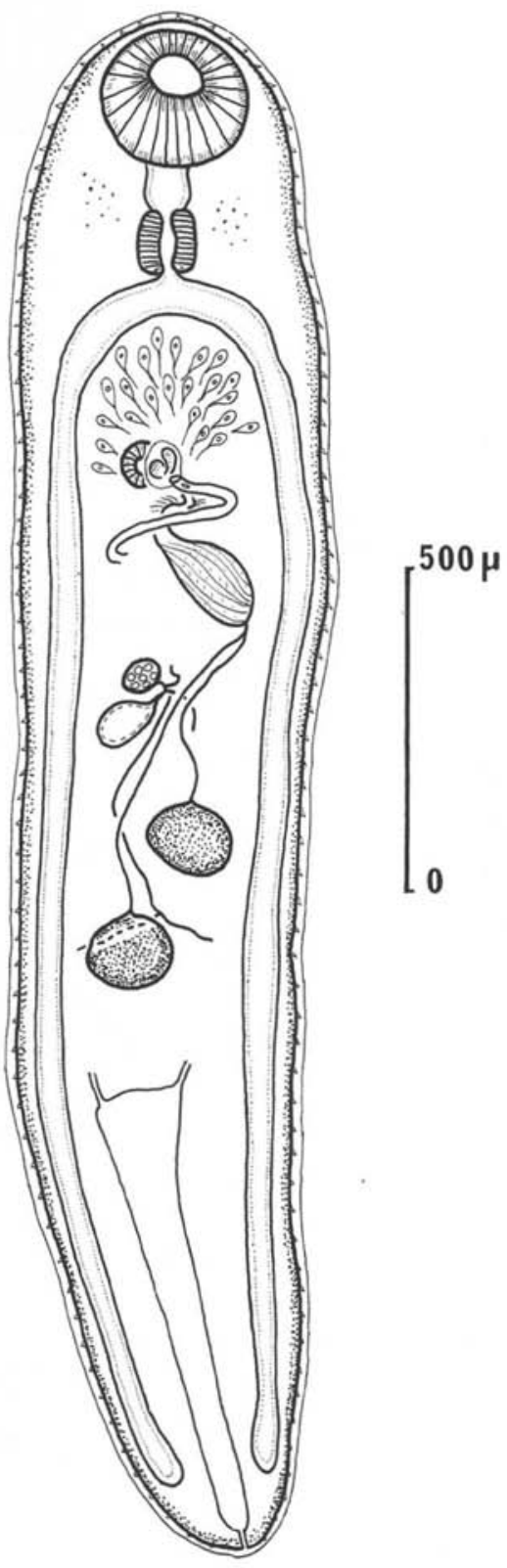

Fig. 4. - Métacercaire mûre de G. timon. davidi. Vue ventrale provenant des trois autres groupes de cellules. L'ensemble de ces canaux formera le 


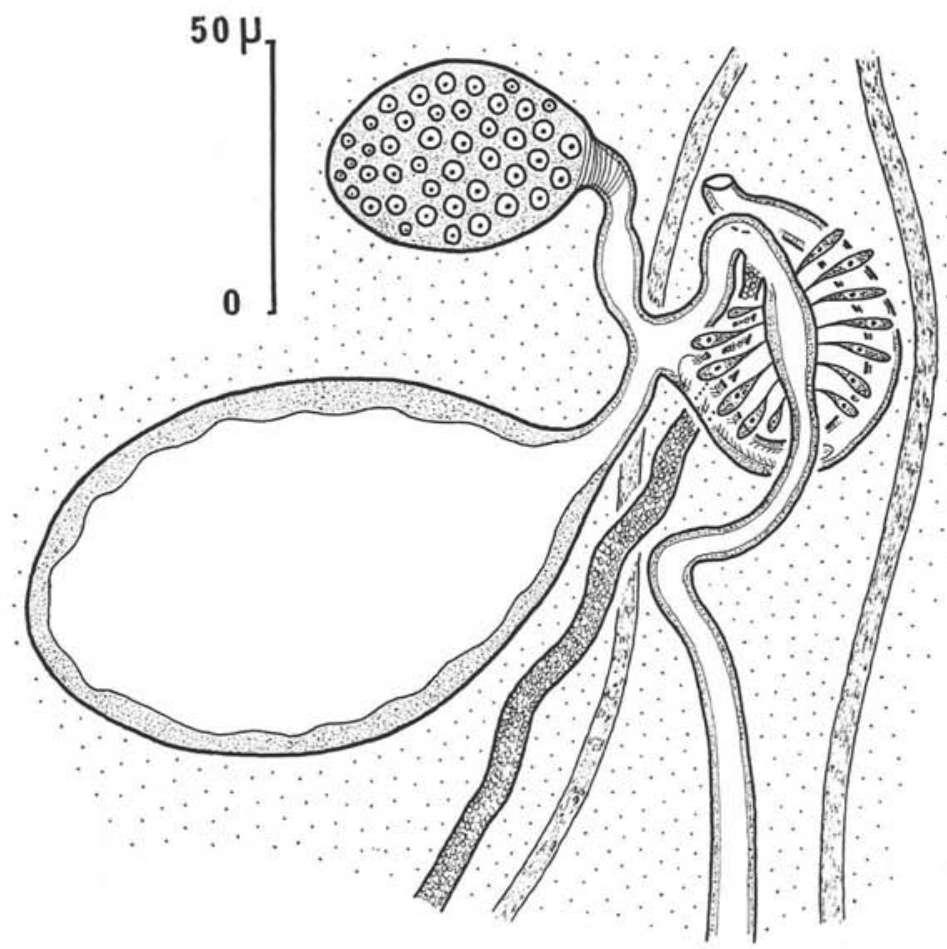

Fig. 5. - Métacercaire de G. timondavidi : carrefour vitellin. Vue ventrale
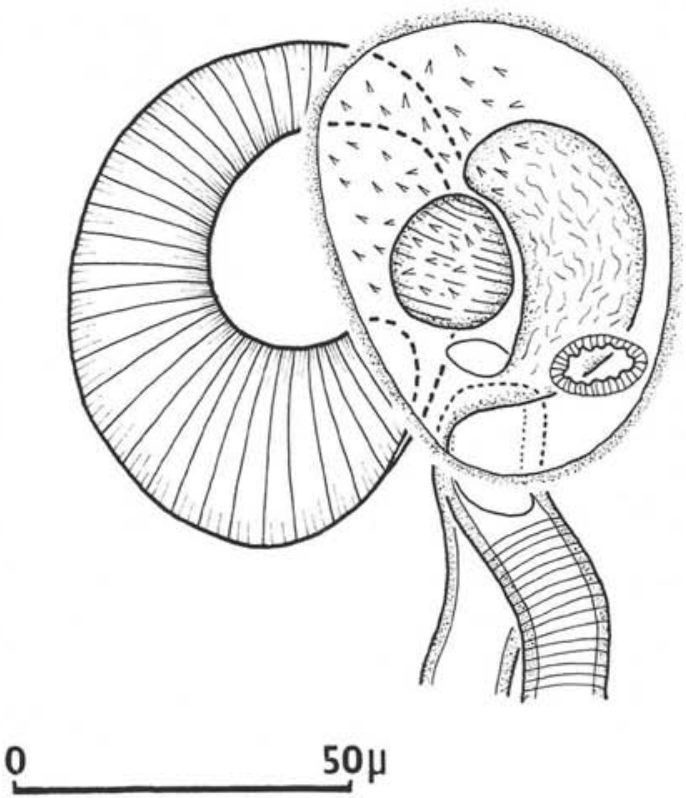

FIG. 6. - Métacercaire de G. timondavidi: complexe ventro-génital. Vue ventrale 


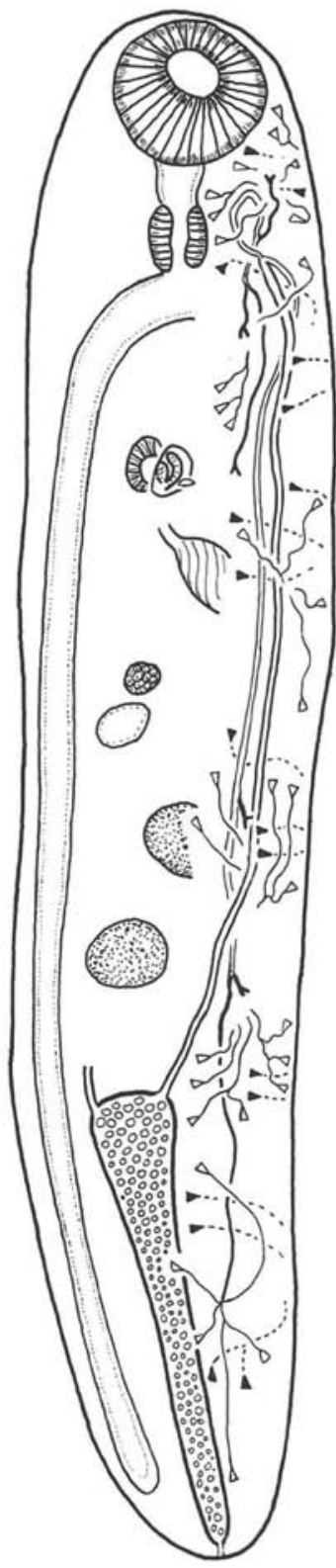

Fig. 7. - Métacercaire de G. timondavidi : appareil excréteur

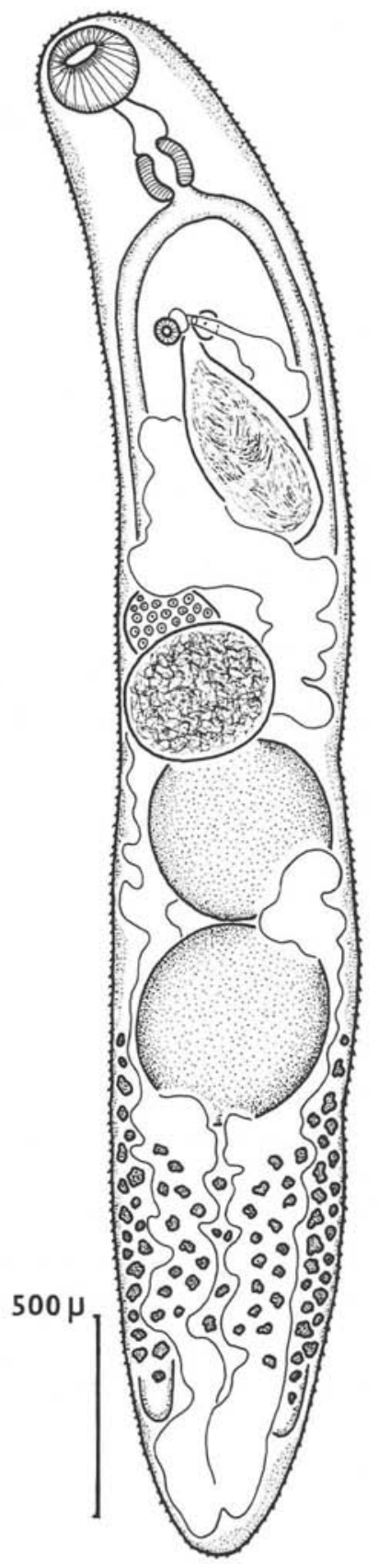

Fig. 8. - G. timondavidi. Adulte de l'intestin de Larus argentatus. Vue ventrale. 
canal collecteur principal. Les deux canaux collecteur principaux s'abouchent à la partie antéro-latérale de la vessie tubuleuse de $350-650 \times 72-80 \mu$. Le pore excréteur est terminal.

Nous avons, après Rebecq et Leray (1960) et Timon-David (1970), retrouvé cette cercaire à l'état naturel dans le cerveau de Diplodus annularis L. (1). En réalité, dans la nature, la cercaire s'enkyste avec la même localisation chez plusieurs autres poissons. Ce sont: Mugil auratus Risso, Syngnathus abaster Risso, Gobius microps Kroyer et Blennius pavo Risso.

Parmi ces hôtes, deux sont pélagiques: ce sont Mugil auratus et Syngnathus abaster. Très probablement, ce sont eux qui jouent un rôle prépondérant dans la réalisation du cycle, du fait qu'ils sont plus accessibles pour l'hôte définitif.

\section{$4^{\circ}$ Adulte (fig. 8).}

Nous l'avons trouvé à la limite du $2^{\circ}$ et du $3^{\circ}$ tiers intestinal de Goélands préalablement infestés à partir de métacercaires provenant de Muges. Ces poissons furent eux-mêmes parasités au laboratoire par des cercaires.

Nous avons en outre retrouvé, par trois fois, l'adulte naturel chez des Goélands de Port-Saint-Louis.

Nous renvoyons pour sa description au travail de Pearson et Prévot (1971).

\section{Résumé du cycle.}

Il se réalise de la façon suivante :

- développement de rédies dans la glande digestive du Gastéropode Cerithium mediterraneum;

- formation de cercaires à l'intérieur des rédies, puis ponte et maturation de ces cercaires dans les tissus de l'hépato-pancréas du Mollusque;

- la cercaire, libre dans l'eau de mer, pénètre chez divers Poissons, principalement les deux espèces pélagiques suivantes: Mugil auratus et Syngnathus abaster;

- maturation de la métacercaire au niveau des lobes optiques du $2^{\circ}$ hôte ;

- l'adulte se développe dans le tube digestif du Goéland Larus argentatus; ce dernier s'infeste en mangeant des poissons parasités.

(1) J. Timon-David et P. Timon-David (1970), se basant sur une étude comparée, avaient attribué la métacercaire du cerveau de Diplodus à la larve de Knipowitschetrema echinatum J. TimonDavid, 1955. L'organisation du complexe ventrogénital et le développement expérimental de cette métacercaire montrent qu'il s'agit en fait de la larve de Galactosomum timondavidi. 


\section{Bibliographie}

CABle (R. M.), 1956. - Scientific Survey of Puerto Rico and the Virgin Islands. Marine Cercariae of Puerto Rico. N.Y. Acad. Sc., 16, 490-577.

-, 1963. - Marine cercariae from Curaçao and Jamaica. Z. f. Parasitenkunde, 23, 429-469.

ITo (J.), 1956. - Studies on the brackish water cercariae in Japan. II. Two new longtailed cercariae, Cercaria komiyai n.sp. and Cercaria nigro caudata n.sp. in Tokyo Bay (Trematoda). Jap. Jour. Med. Sci. and Biol., 9, 235-242.

Joyeux (Ch.) et BAER (J.-G.), 1928. - Note sur quelques Helminthes récoltés en Macédoine. Bull. Soc. Path. Exot. Paris, 21, 214-220.

Miller (H. M.), 1925. - A preliminary report of the larval Trematodes infesting certain Mollusks from Dry Tortugas. Carnegie Inst. Year Book, 24, 232-238.

- 1929. - Continuation of study on behavior and reactions of marine cercariae from Tortugas. Carnegie Inst. Year Book, 28, 292-294.

Monticelli (F.S.), 1888. - Saggio di una morfologia dei trematodi. Tesi per ottenere la privata docenza in Zoologie nella $R$. Universita di Napoli, $130 \mathrm{p}$.

Pearson (J. C.) et Prévot (G.), 1971. - Galactosomum timondavidi sp.n. (Trematoda: Heterophyidae) from Larus argentatus, with a note on the metacercaria. J. Parasit., $57,1227-1230$.

PrÉvot (G.), 1967. - Contribution à l'étude des cercaires de Prosobranches de la région marseillaise : Cercaria mirabilicaudata n. sp. (Trematoda, Digenea, Opisthorchioidae) de Cerithium vulgatum Brug. Bull. Soc. Zool. France, 92, 515-523.

Rebece (J.) et Leray (C.), 1960. - Observations sur trois Trématodes hébergés par quelques Poissons des calanques marseillaises. Bull. Inst. Océanogr. Monaco, 57, $1190,1-15$.

SINITZIN (D. T.), 1911. - Parthenogenetic generation of trematodes and its progeny in Molluscs of the Black Sea. Rec. Imp. Acad. Sci., St-Petersburg, sér. 8, 30, 127 p.

Timon-David (J.) et Timon-David (P.), 1970. - A study on a larval trematode occuring. in the brain of the marine fish Diplodus annularis (L.). H.D. Srivastava Commen. 283-288. 